\title{
Effectiveness of the postcoital test: randomised controlled trial
}

\author{
S Guid Oei, Frans M Helmerhorst, Kitty W M Bloemenkamp, Frederieke A M Hollants, \\ Debbie E M Meerpoel, Marc J N C Keirse
}

Department of
Obstetrics and
Gynaecology, Saint
Joseph Hospital,
$5500 \mathrm{MB}$
Veldhoven,
Netherlands
S Guid Oei,
gynaecologist
Department of
Obstetrics,
Gynaecology and
Reproductive
Medicine, Leiden
University Medical
Center, PO Box
9600, 2300 RC
Leiden, Netherlands
Frans M
Helmerhorst,
senior lecturer
Kitty W M
Bloemenkamp,
registrar
Frederieke A M
Hollants,
medical officer
Debbie E M
Meerpoel,
medical officer
Department of
Obstetrics and
Gynaecology,
Flinders University
of South Australia,
Adelaide, SA 5042,
Australia
Marc J N C Keirse,
professor
Correspondence to:
Dr Helmerhorst
helmerhorst@rullf2.
medfac.leidenuniv.nl

BMJ 1998;317:502-5

\begin{abstract}
Objectives: To investigate the impact of the postcoital test on the pregnancy rate among subfertile couples and on the number of other diagnostic tests and treatments.

Design: Randomised controlled study.

Setting: A university and two non-university teaching hospitals in the Netherlands.

Subjects: New couples at infertility clinics, 1 March 1993 to 1 October 1995; randomisation to an intervention group (series of infertility investigations that include the postcoital test) or to a control group (series excluding the test).

Main outcome measure: Cumulative pregnancy rate. Results: Of 736 consecutive new couples, 444 fulfilled the inclusion criteria and consented to participate (intervention group, 227; control group, 217). Treatment was given more often in the intervention group than in the control group (54\% v 41\%; difference $13 \%$ (95\% confidence interval $4 \%$ to $22 \%)$ ). Yet cumulative pregnancy rates at 24 months in the intervention group (49\% (42\% to $55 \%)$ ) and the control group ( $48 \%(42 \%$ to $55 \%)$ ) were closely similar (difference 1\% (-9.0\% to $9.0 \%)$ ).

Conclusion: Routine use of the postcoital test in infertility investigations leads to more tests and treatments but has no significant effect on the pregnancy rate.
\end{abstract}

\section{Introduction}

First introduced in $1866,{ }^{1}$ postcoital testing of cervical mucus for the presence of progressively motile sperm has become an important part of infertility investigations. It is used routinely in nearly half of the fertility clinics in Britain and in two thirds of such clinics across Europe. ${ }^{2}$ Yet observational studies show that the diagnostic and prognostic power of the postcoital test is limited ${ }^{3}{ }^{4}$-so is its value for assessing and treating cervical hostility to sperm. ${ }^{2}$ Various treatments are used in cases of abnormal postcoital test results, with intrauterine insemination a clear favourite despite the fact that its effectiveness has not been shown..$^{5-8}$ Theoretically, however, it is possible to find a significant effect of treatment even if it is based on a test with poor diagnostic and prognostic properties. Against this background we conducted a randomised controlled trial comparing infertility investigations with and without postcoital testing to assess the usefulness of such testing for routine fertility investigations.

\section{Methods}

We compared a series of infertility investigations that routinely included the postcoital test (intervention group) and a series of investigations that excluded the test unless clinicians felt a definite need for it (control group). Cumulative pregnancy rates were the primary outcome of interest. The secondary outcome was the number of other diagnostic tests and treatments in the two groups. To avoid bias clinicians were not told about these secondary outcomes. Apart from the postcoital test, they were free to apply whatever diagnostic tests and treatments they considered to be appropriate.

\section{Patients and randomisation procedure}

From 1 March 1993 to 1 October 1995, subfertile couples attending the reproductive medicine units at Leiden University Hospital, Westeinde Hospital (The Hague), and Groene Hart Hospital (Gouda) were candidates for participation in the study. To avoid bias at all subsequent stages, a sequentially numbered, opaque, sealed envelope was assigned to each woman's case notes before she was seen or as soon as a fertility problem was presented. Couples were excluded if the woman had been referred for in vitro fertilisation, donor insemination, intrauterine insemination, or tubal surgery or if she had any health problem unrelated to fertility. If the exclusion criteria did not apply, informed consent was requested. Envelopes for women who were excluded or did not consent were returned to the central randomisation office and discarded unopened.

The study protocol was approved by the institutional review boards of the hospitals. It foresaw predicted a 1:1 randomisation of women to the intervention and control groups.

\section{Postcoital test procedure}

The postcoital test was planned 14-16 days before menstruation and 6-18 hours after intercourse. We asked the couples to have intercourse as usual on the night before the test; we gave no instructions on prior abstinence, postcoital rest, or posture. A non-lubricated speculum was used to expose the cervix. Endocervical mucus was collected by suction in a narrow $1 \mathrm{ml}$ disposable syringe. Mucus was considered to be good if it was abundant $(\geqslant 0.3 \mathrm{ml})$, highly ductile $(\geqslant 100 \mathrm{~mm})$, and mostly clear to the naked eye.

The number of sperms moving forward per high power field ( $\times 400$ magnification) was recorded. A negative or abnormal ("absent" or "non-motile") sperm result was considered valid only if the mucus was in good condition or if the test had been repeated and timed by ultrasonic measurement of follicles and serum oestradiol concentration in a later cycle. Positive or normal results were accepted whatever the state of the mucus.

\section{Infertility treatments}

Treatment for negative postcoital test results was in accordance with standard clinical practice. ${ }^{2}$ Intrauterine insemination was performed with the aid of ovarian stimulation with $100 \mathrm{mg}$ clomiphene citrate 
daily from days 3 to 7 of the cycle, followed by injection of $10000 \mathrm{IU}$ human chorionic gonadotrophin (timed by cycle monitoring). This was followed, 40-44 hours later, by intrauterine insemination of sperm after sperm collection with a discontinuous Percoll gradient method. In vitro fertilisation was performed as described by Van de Berg et al..$^{9}$ Artificial insemination with donor semen was performed after luteinised hormone detection in urine (Ovuquick, Quidel, San Diego, CA) in a natural cycle.

Diagnosis of pregnancy was based on a positive pregnancy test in urine (Pregstik 50/1000, Nourypharma, Oss, Netherlands) 15 days or more after ovulation.

\section{Statistical methods}

Descriptive statistics were used to assess the similarity of the groups. Cumulative pregnancy rates were calculated as for life table analysis and compared with the Gehan-Wilcoxon test. Categorical data, including cumulative pregnancy rates at 24 months, were assessed by the $\chi^{2}$ test and continuous variables by Student's $t$ test $(\mathrm{P}<0.05$ was considered significant). With the smallest difference in success rate set at $10 \%$ (with an $\alpha$ error of 0.05 and a $\beta$ error of 0.20 ), 350 couples were required in each arm.

\section{Results}

Of 736 consecutive couples with presumed infertility, 287 were excluded according to the criteria, and 5 did not consent (table 1). The remaining 444 couples were randomised to the intervention $(\mathrm{n}=227)$ and control ( $\mathrm{n}=217)$ groups. The characteristics of the participants did not differ significantly between the two groups (table $2^{2}$ ).

Follow up either to pregnancy or for 24 months was complete in both groups. At the end of the study the postcoital test had been performed in 146 of the $227(64 \%)$ couples in the intervention group, with an average frequency of 1.3 times (range 1-4) per couple. Reasons for not performing a postcoital test were pregnancy $(\mathrm{n}=57)$, anovulation $(\mathrm{n}=9)$, bilateral tubal occlusion $(n=9)$, or azoospermia $(n=6)$. In the control group 3 of the $217(1 \%)$ couples had undergone a postcoital test.

Table 3 shows the infertility investigations performed in both groups. Hysterosalpingography was used in $114(50 \%)$ women in the intervention group and in $117(54 \%)$ in the control group. Thirty two (14\%) women in the intervention group and $44(20 \%)$ in the control group underwent laparoscopy. Hysterosalpingography was performed 4.7 (range 2.2-8.3)

Table 1 Reasons for exclusion of 292 women from study after sealed envelopes were assigned

\begin{tabular}{lc} 
Reason for exclusion & No of women \\
\hline Had been referred for in vitro fertilisation & 67 \\
\hline Had been referred for artificial insemination & 92 \\
\hline Had been referred for tubal operation & 4 \\
\hline Had problems unrelated to infertility & 94 \\
\hline Was pregnant at first visit & 30 \\
\hline Did not give informed consent & 5 \\
\hline
\end{tabular}

*Patients with endocrine problems, with questions on future fertility after exposure to diethylstilboestrol in utero, radiotherapy, chemotherapy, primary dysmenorrhoea etc.
Table 2 Characteristics of couples participating in study

\begin{tabular}{lcc} 
Characteristics & Intervention group (n=227) & Control group ( $\mathbf{n = 2 1 7 )}$ \\
\hline Mean (range) age of woman (years) & $30.8(18.8-41.0)$ & $30.4(19.4-43.6)$ \\
\hline Mean (range) age of man (years) & $33.5(22.8-56.4)$ & $32.6(22.5-57.7)$ \\
\hline Mean (range) duration of infertility (months) & $26.7(12-480)$ & $24.6(12-144)$ \\
\hline No (\%) of couples with history of sexual problems & $35(15)$ & $27(12)$ \\
\hline No (\%) of partners with sperm disorder & $72(32)$ & $72(33)$ \\
\hline No (\%) of women with ovulatory disorder & $50(22)$ & $38(18)$ \\
\hline No (\%) of women with tubal disorder & $20(9)$ & $27(12)$ \\
\hline
\end{tabular}

Table 3 Number (percentage) of infertility investigations performed in intervention group and control group

\begin{tabular}{lcc} 
Investigation & Intervention group $(\mathbf{n}=227)$ & Control group $(\mathbf{n}=\mathbf{2 1 7})$ \\
\hline Postcoital test & $146(64)$ & $3(1)$ \\
\hline In vitro cervical mucus test & $17(7)$ & $1(0.4)$ \\
\hline Semen analysis & $219(96)$ & $201(93)$ \\
\hline Hysterosalpingography & $114(50)$ & $117(54)$ \\
\hline Laparoscopy & $32(14)$ & $44(20)$ \\
\hline
\end{tabular}

Table 4 Numbers (percentages) of treatments given in intervention group and control group

\begin{tabular}{lcc} 
Treatment & $\begin{array}{c}\text { Intervention group } \\
(\mathbf{n}=\mathbf{2 2 7})\end{array}$ & $\begin{array}{c}\text { Control group } \\
(\mathbf{n}=217)\end{array}$ \\
\hline Intrauterine insemination & $49(22)$ & $36(17)$ \\
\hline Bicarbonate irrigation & $3(1)$ & $1(0.5)$ \\
\hline In vitro fertilisation & $22(10)$ & $14(6)$ \\
\hline Artificial insemination donor & $1(0.4)$ & $1(0.5)$ \\
\hline Ovulation induction & $42(19)$ & $35(16)$ \\
\hline Other treatment & $5(2)$ & $2(1)$ \\
\hline Total & $122(54)$ & $89(41)^{\star}$
\end{tabular}

${ }^{*} \chi^{2}$ test $=7.21(\mathrm{df}=1 ; \mathrm{P}<0.05)$

months after the first visit in the intervention group and 3.8 (2.1-7.8) months after the first visit in the control group. The corresponding time intervals for laparoscopy after the first visit were 8.3 (3.4-13.2) months and 6.6 (3.3-12.8) months.

Table 4 shows the treatments for infertility in both groups. Intrauterine insemination was performed in 49 $(22 \%)$ women in the intervention group, starting 8.4 (3.6-18.3) months after the first visit and in $36(17 \%)$ in the control group, starting 8.6 (3.7-19.2) months after the first visit. Indications for intrauterine insemination in the control group were low sperm count and unexplained infertility. Twenty two (10\%) couples in the intervention group and $14(6 \%)$ in the control group underwent in vitro fertilisation. Overall, 122 (54\%) couples in the intervention group received treatment, compared with 89 (41\%) in the control group, a difference of $13 \%$ (95\% confidence interval $4 \%$ to $22 \%)$.

The cumulative pregnancy rate at 24 months was $48 \%(42 \%$ to $55 \%)$ in the intervention group and $49 \%$ ( $42 \%$ to $55 \%$ ) in the control group, a difference of $1 \%$ (-9\% to $9 \%$ ). Pregnancy rates at given times throughout study did not differ significantly either (figure). Analysis based on the couples in the intervention group who underwent the postcoital test and on those in the control group who did not have a postcoital test showed a significantly lower pregnancy rate in the intervention group $(53 / 146(36 \%))$ than in the control group $(105 / 214(49 \%))$.Although this represents a difference of $13 \%$ (3\% to $23 \%$ ), the difference is partially due to those conceiving before the postcoital test had been performed. 


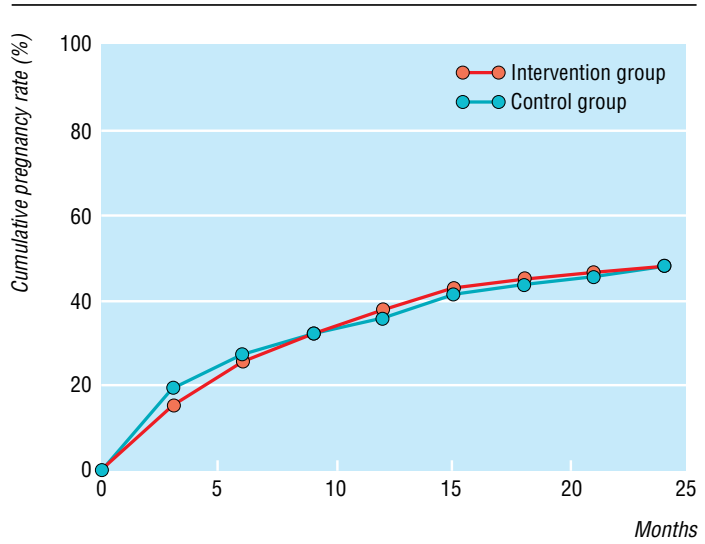

Cumulative pregnancy rates for 227 couples in intervention group (which included postcoital test) and 217 couples in control group (which excluded the test)

Of the 146 women in the intervention group who underwent the postcoital test, 79 had a normal result, of whom $30(38 \%)$ became pregnant; of the 67 women who had an abnormal result, 23 (34\%) conceived, a difference of only $4 \%(-19 \%$ to $12 \%)$. Of the three control women who underwent a postcoital test, one had a normal result and two an abnormal one; none of them conceived.

\section{Discussion}

We conducted a randomised study to assess the usefulness of the postcoital test in investigating infertility. Because the usefulness of the test was uncertain, ${ }^{2}{ }^{10}$ we justified excluding it from the standard series of infertility investigations. This made it possible to conduct a randomised comparison of two infertility protocols that were identical except for inclusion and exclusion of the postcoital test.

\section{Intention to treat principle}

Although 190 postcoital tests were performed in 146 couples in the intervention group, $36 \%$ of the $227 \mathrm{cou}-$ ples in that group did not undergo the test. To deal with this and the fact that three couples underwent a postcoital test in the control group, our analysis was based on the groups as randomised, following the "intention to treat" principle.

Original power calculation envisaged 350 couples in each group. In the middle of 1995 changes in staff were foreseen that would affect the daily trial management and the enrolment of couples, and therefore the consistency and reliability of the study. Consequently, we ended enrolment on 1 October 1995, thereby accepting a lower power for the study. By that time 444 subfertile couples were participating, each group containing 130 less than the original power calculations required. Given the absolute equality of a close to $50 \%$ pregnancy rate in both groups, a pregnancy rate of about $75 \%$ would have been required in the next 130 women in the intervention group to achieve overall significance at the 5\% level. The chances of this happening were very small, given a success rate less than $50 \%$ in the first 220 women. As the binomial probability of such an occurrence is less than 1 in 100 , it is extremely unlikely that continuing the study would have altered the conclusions that can be drawn from it.

More treatments but no effect on pregnancy rate Except for in vivo and in vitro testing of the interaction between cervical mucus and semen-which are closely linked to the postcoital test (table 3)-there were no significant differences in other infertility investigations between the intervention and control groups. Although the results show that the intervention group underwent more treatments than the control group (table 4), the cumulative pregnancy rates at 24 months were closely similar in the two groups. Routine use of the postcoital test in infertility investigations seemed to lead to a larger number of treatments with no effect on the cumulative pregnancy rate.

\section{Poor predictor of fertility}

In this study the postcoital test was a poor predictor of fertility. In the intervention group 38\% of women with a normal test result conceived, compared with $34 \%$ of those with an abnormal result, a difference that is not significant despite the treatments given for abnormal results. These poor test properties are consistent with the findings of meta-analyses and systematic reviews published on this subject. ${ }^{34}$

\section{Conclusion}

The simple availability of a test, fear of missing a crucial diagnosis, and simple zeal for the attainment of diagnostic certainty are all inducements to test what can be tested. ${ }^{11-13}$ For both clinical and economic reasons it may be wise, however, for every test to be preceded by a conscious decision to test, based on solid evidence that the test is needed. Good arguments exist, too, for not using tests that offer little chance of changing the scope of diagnostic possibilities. ${ }^{14}$ On the basis of both systematic review of its test properties ${ }^{34}$ and this randomised trial, it is difficult to justify the postcoital test as an essential procedure in standard infertility investigations.

We thank Professor J P Vandenbroucke for critical reading and advice.

Contributors: SGO initiated and designed the study, reviewed the literature, supervised recruitment at Westeinde and

\section{Key messages}

- The postcoital test dates back to 1866

- The test is widely used in infertility investigations but has limited diagnostic potential and poor predictive value

- Treatments for abnormal test results vary but have not been shown to be effective

- This first randomised controlled trial comparing infertility investigations with and without the postcoital test showed closely similar cumulative pregnancy rates at 24 months

- Incorporation of the postcoital test in standard infertility investigations increases the number of tests and treatments but has no effect on the pregnancy rate 
Groene Hart Hospitals, oversaw data collection, analysed data, and jointly wrote the paper with FMH, KWMB, and MJNCK. FMH initiated and designed the study, supervised recruitment at Leiden University Hospital, supervised data collection, and analysed data. KWMB helped to develop the trial and collect data, and analysed data. FAMH and DEMM were responsible for completing data sheets and data entry. Marc Keirse helped in the study design; was responsible for power calculation, central randomisation, and termination of enrolment; reviewed data; and edited the paper.

Funding: Financial support was received from the participating institutions only.

Conflict of interest: None

1 Sims JM. Clinical notes on uterine surgery (with special reference to the management of the sterile condition). London: Robert Hardwicke, 1866.

2 Oei SG, Keirse MJNC, Bloemenkamp KWM, Helmerhorst FM. European postcoital tests: opinions and practice. $\mathrm{Br} J$ Obstet Gynaecol 1995;102:621-4

3 Griffith CS, Grimes DA. The validity of the postcoital test. Am J Obstet Gynecol 1990;162:615-20.

4 Oei SG, Helmerhorst FM, Keirse MJNC. When is the postcoital test normal: a critical appraisal. Hum Reprod 1995;10:1711-4.

5 Glazener CMA, Coulson C, Lambert PA, Watt EM, Hinton RA, Kelly NJ, et al. The value of artificial insemination with husband's semen in infertility due to failure of postcoital sperm-mucus penetration-controlled trial of treatment. BrJ Obstet Gynaecol 1987;94:774-8.
6 Te Velde ER, van Kooy RJ, Waterreus JJH. Intrauterine insemination of washed husband's spermatozoa: a controlled study. Fertil Steril $1989 ; 51: 182-5$.

7 Friedman A, Haas S, Kredentser J, Stewart E, Schiff I. A controlled trial of intrauterine insemination for cervical factor and male factor: a preliminary report. Int J Fertil 1989;34:199-203.

8 Kirby CA, Flaherty SP, Godfrey BM, Warnes GM, Matthews CD. A prospective trial of intrauterine insemination of motile spermatozoa versus timed intercourse. Fertil Steril 1991;56:102-7.

9 Van de Berg-Helder A, Helmerhorst FM, Blankhart A, Brand R, Waegemaekers C, Naaktgeboren N. Comparison of ovarian stimulation regimens for in vitro fertilization (IVF) with and without a gonadotropinreleasing hormone (GNRH) agonist: results of a randomized study. J Vitro Fertil Embryo Transfer 1990; 7:358-62.

10 Rowe PJ, Comhaire FH, Hargreave TB, Mellows HJ. WHO manual for the standardized investigation of the infertile couple. Cambridge: Cambridge University Press, 1993.

11 Allman RM, Steinberg EP, Keruly JC, Dans PE. Physician tolerance for uncertainty: use of liver-spleen scans to detect metastases. JAMA $1985 ; 254: 246-8$.

12 Eisenberg JM, Schumacher HR, Davidson PK, Kaufmann L. Usefulness of synovial fluid analysis in the evaluation of joint effusions: use of threshold analysis and likelihood ratios to assess a diagnostic test. Arch Intern Med 1984;144:715-9.

13 Thibault GE. The appropriate degree of diagnostic certainty. N Engl J Med 1994;331:1216-20.

14 Putterman C, Ben-Chetrit E. Clinical problem solving: testing, testing, testing. . . . N Engl J Med 1995;333:1208-11.

(Accepted 13 May 1998)

\section{Does moderate alcohol consumption affect fertility? Follow up study among couples planning first pregnancy}

Tina Kold Jensen, Niels Henrik I Hjollund, Tine Brink Henriksen, Thomas Scheike, Henrik Kolstad, Aleksander Giwercman, Erik Ernst, Jens Peter Bonde, Niels E Skakkebæk, Jørn Olsen

\begin{abstract}
Objective: To examine the effect of alcohol consumption on the probability of conception. Design: A follow up study over six menstrual cycles or until a clinically recognised pregnancy occurred after discontinuation of contraception.

Subjects: 430 Danish couples aged 20-35 years trying to conceive for the first time.

Main outcome measures: Clinically recognised pregnancy. Fecundability odds ratio: odds of conception among exposed couples divided by odds among those not exposed.

Results: In the six cycles of follow up 64\% (179) of women with a weekly alcohol intake of less than five drinks and $55 \%$ (75) of women with a higher intake conceived. After adjustment for cycle number, smoking in either partner or smoking exposure in utero, centre of enrolment, diseases in female reproductive organs, woman's body mass index, sperm concentration, and duration of menstrual cycle, the odds ratio decreased with increasing alcohol intake from $0.61(95 \%$ confidence interval 0.40 to 0.93) among women consuming 1-5 drinks a week to 0.34 (0.22 to 0.52 ) among women consuming more than 10 drinks a week $(\mathrm{P}=0.03$ for trend) compared with women with no alcohol intake. Among men no dose-response association was found after control for confounders including women's alcohol intake.

Conclusion: A woman's alcohol intake is associated with decreased fecundability even among women with a weekly alcohol intake corresponding to five or fewer
\end{abstract}

drinks. This finding needs further corroboration, but it seems reasonable to encourage women to avoid intake of alcohol when they are trying to become pregnant.

\section{Introduction}

The incidence of infertility is high and expected to increase. Intake of alcohol is a possible causal factor of public health importance as consumption is widespread and increasing in many countries. In experimental animals alcohol is known to decrease steroid hormone concentrations, inhibit ovulation, and interfere with sperm cell transportation through the fallopian tube. ${ }^{1}$ Alcohol given to rats and monkeys reduces ovarian weight and causes amenorrhoea. ${ }^{23}$

The concentration of sulphated steroids has been found to be lower in alcoholic women than in controls. ${ }^{3}{ }^{4}$ Furthermore, chronic alcohol misuse in women has been associated with changes in hepatic oestrogen receptors. ${ }^{5}$ Women with high or frequent alcohol intake have been found to have higher rates of menstrual disorders, including amenorrhoea, dysmenorrhoea, and irregular menstrual periods ${ }^{6-8}$ Pregnant women with a high alcohol intake have a higher incidence of miscarriages, placental abruption, preterm deliveries, and stillbirths than control women. ${ }^{6}$ Alcohol in high doses is also known to be teratogenic and is responsible for fetal alcohol syndrome..$^{10}$ The effect of moderate alcohol intake on reproduction, however, is less well examined.

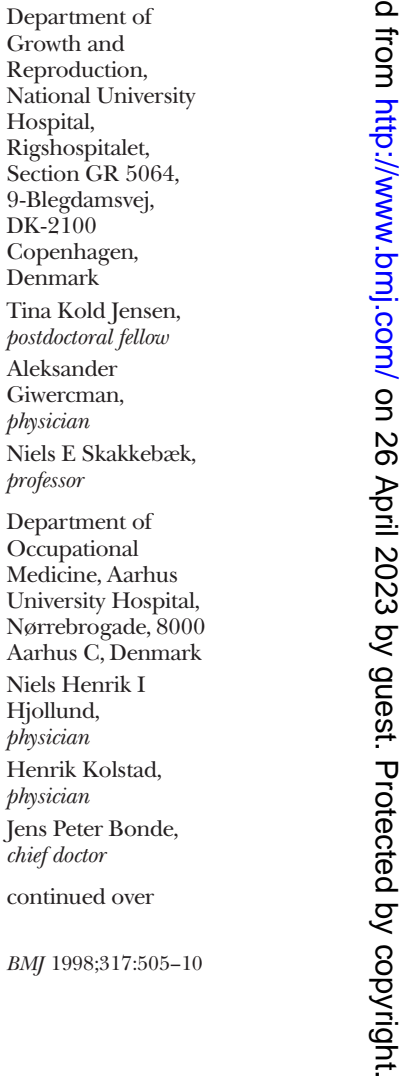

